\title{
Intención de compra de productos verdes de acuerdo con la Teoría del Comportamiento Planeado: Incorporación de la obligación moral al modelo
}

\section{Intention to purchase green products according to the Theory of Planned Behaviour: Incorporation of the moral obligation to the model}

Jessica Müller Pérezi*

Universidad Autónoma de Tamaulipas

jsmuller@docentes.uat.edu.mx

Juan Bernardo Amezcua Núñez

Universidad Autónoma de Coahuila, Saltillo

juan.amezcua@uadec.edu.mx

Sheyla Müller Pérez

Universidad Autónoma de Chile

sheyla.muller@uautonoma.cl
* Autor corresponsal.

Facultad de Comercio y Administración Victoria. Cd.Victoria, Tamaulipas, MÉXICO.

ii Facultad de Mercadotecnia, Universidad Autónoma de Coahuila. Saltillo, Coahuila. MÉXICO.

iii Universidad Autónoma de Chile. Santiago, CHILE.

\section{Resumen}

El comportamiento de compra del consumidor verde ha ido incrementando en los últimos años. De acuerdo con Kantar World Panel México en 2012, solo el $16 \%$ de los mexicanos realizaba alguna acción ecológica y, en el 2019, 7 de cada 10 mexicanos optan por productos ecológicos. El estudio tiene por objetivo identificar qué variables del modelo de la Teoría del Comportamiento Planeado realmente afectan la intención de compra de productos verdes en los jóvenes y, demostrar que la variable de obligación moral debe incluirse en dicho modelo. Se aplicó una encuesta en línea a un grupo de 280 estudiantes de escuelas públicas de la región Noreste de México. Los datos fueron analizados a través del modelo de ecuaciones estructurales con mínimos cuadrados parciales (PLS-SEM), el cual reveló que solo la actitud y la obligación moral tienen un efecto positivo y significativo hacia la intención de compra de productos verdes

\begin{abstract}
Green consumer buying behavior has recently increased. According to Kantar World Panel Mexico in 2012, only 16\% of Mexicans carried out some ecological action and, in 2019, 7 of 10 Mexicans are willing to buy ecological products. In order to assess the motivators who have driven this change this study aims to examine the intention to purchase green products on young consumers based on the Theory of Planned Behavior incorporating moral obligation. An online survey was applied to a group of 280 public school students from the Northeast region of Mexico. The data were analyzed through the structural equations model with partial least squares (PLS-SEM), which revealed that only attitude and moral obligation have a positive and significant effect on the intention to buy green products.
\end{abstract}

INFORMACIÓN ARTÍCULO

Recibido: 30 de Junio 2020

Aceptado: 16 de Noviembre 2020
Palabras Claves:

Teoría del comportamiento planeado Productos verdes

Intenciones

Obligación moral
Keywords:

Theory of planned behavior

Green products

Intentions

Moral values 


\section{INTRODUCCIÓN}

Hoy día el tema de sustentabilidad o protección al medio ambiente ha recibido la atención de la sociedad en muchos países del mundo, además, de ir en aumento conforme a los problemas de contaminación, falta de recursos naturales y a la extinción de especies, los cuales han sido estudiados en la última década (Nguyen y cols., 2016). Sin embargo, las empresas participan en una conducta ambiental responsable dentro de sus actividades administrativas (Szabo y Webster, 2020). Lo anterior, en respuesta a las preocupaciones ambientales que los consumidores han expuesto en años recientes, a través de la adquisición de productos que ayuden a disminuir el daño (Paul y cols., 2016)a critical variable in green marketing literature, intending to achieve triple bottom line (TBL. Y, por lo tanto, las personas con creencias y actitudes más favorables hacia el cuidado del medio ambiente tienen mayores intenciones de comprar productos verdes (Szabo y Webster, 2020). Es por esto, que, es de suma importancia identificar qué variables del modelo de la Teoría del Comportamiento Planeado (TCP), tienen mayor peso en cuanto a la formación del consumidor comprometido con el medio ambiente en el momento de tomar sus decisiones de compra y consumo; todo esto, con el propósito de desarrollar y ampliar la oferta de mercado sensible a las necesidades y los deseos de dichos consumidores (Castañeda, 2014).

Por su parte, Chan y Bishop (2013), mencionan que existen estudios previos que enfatizan en agregar al modelo de la TCP la variable de obligación moral, argumentando que tanto la obligación moral y las actitudes comparten correlaciones muy altas, puesto que la obligación moral ha sido considerada como un antecedente poderoso de las actitudes hacia el cuidado del medio ambiente. De igual manera, Chen (2020), expone que la obligación moral puede incrementar el poder predictivo del modelo de la TCP en el contexto de productos amigables con el medio ambiente. En tanto, Beldad y Hegner (2018), dedujeron que la adición de la obligación moral al modelo, aumentaba la varianza explicada en la intención de consumir productos verdes. En este sentido, es necesario resaltar que la falta de respuesta por parte de los consumidores en dicho contexto, es por no considerar que los problemas ambientales son un problema de índole moral (Chan y Bishop, 2013). Asimismo, se estudian los factores de la
TCP agregando la variable de obligación moral en el contexto de intención de compra de productos verdes.

Como primer objetivo, este estudio pretende contribuir a la literatura de intención de compra en el contexto de productos verdes, basándose en la TCP, y a pesar de que dicha teoría ha sido muy criticada, sigue siendo aún relevante y necesaria para comprender el comportamiento humano (Ajzen, 2015). Segundo, aportar conocimiento en cuanto a los factores de la TCP que más afectan en la intención de compra de los jóvenes consumidores mexicanos del estado de Tamaulipas, siendo un grupo generacional muy poco estudiado en el contexto de productos verdes (Su y cols., 2019). $\mathrm{Y}$, tercero, demostrar que la dimensión de obligación moral debe agregarse en el modelo de la TCP en el contexto del consumidor ecológico mexicano

\section{REVISIÓN DE LA LITERATURA Y MÉTODO DE INVESTIGACIÓN}

\section{Intención de compra de productos verdes}

La intención de compra de un consumidor se ha conceptualizado en la probabilidad de que compre o planee adquirir cierto producto o servicio en un futuro próximo, por lo que, un aumento en la intención de compra es el reflejo de un aumento en la posibilidad de compra (Martins y cols., 2019). De acuerdo con Schill y cols. (2019), Ia TCPP aplicado al consumo ecológico, supone que la intención del consumidor de realizar un comportamiento responsable es determinado por ciertas variables, tales como: las actitudes, las nomas subjetivas y el control de compra percibido. Sin embargo, para el propósito de este estudio se agrega la variable de obligación moral al modelo propuesto por Ajzen (1991).

De acuerdo con Rashid (2009), la intención de compra de productos amigables con el medio ambiente se percibe como la disposición de una persona de adquirir productos verdes, en lugar de productos convencionales. En ese mismo sentido, Zhikun y Fungfai (2009), mencionan que las intenciones corresponden al predictor más importante del comportamiento humano, y los humanos son racionales en el uso sistemático de la información disponible.

Con respecto a los productos verdes, estos son considerados como aquellos productos que con- 
tienen uno o más componentes amigables con el medio ambiente, en otras palabras, producen menos contaminación, son renovables y, sobre todo, menos dañinos para el medio ambiente (Nguyen y cols., 20; Spielmann, 2020). Algunos productos verdes son hechos a base de papel, vidrio (envases reciclables o reutilizables), dispositivos de ahorro de energía y detergentes que deben ser biodegradables y sin contaminación (Mostafa, 2006). Debido a eso, la intención de un consumidor en participar de un comportamiento bajo ciertas consideraciones como la adquisición de productos verdes, es considerado como precursor y el mejor predictor del comportamiento humano (Han y Kim, 2010). Significa, entonces, que una intención de compra de productos verdes indica el grado en el cual los consumidores están dispuestos o listos para comprar productos amigables con el medio ambiente, o por lo menos, adoptar opciones más ecológicas (Ajzen, 2002).

De acuerdo con Larios-Gómez (2019), 8 de cada 10 mexicanos están preocupados por el cuidado del medio ambiente y el $60 \%$ de ellos asegura que si un producto es ecológico influirá en su decisión de compra. Sin embargo, en un estudio realizado por Green Study of Research International México' (2014), se encontró que solo el 50\% de los mexicanos está dispuesto a comprar productos amigables con el medio ambiente, porque existe poca disponibilidad para adquirir dichos productos y los precios son más altos en comparación con los productos tradicionales (Larios-Gómez, 2019). Además, un estudio reciente de Worldpanel de Kantar México (2020), señaló que a partir del impacto que el COVID-19 trajo a los mexicanos, obligándolos a mantenerse en casa, el cielo se limpió y los animales se sintieron libres de pasear en las ciudades, por lo que los ciudadanos adoptaron acciones más amigables con el medio ambiente incrementando algunas acciones, por ejemplo: aumento del uso de bolsas ecológicas de un 33\% a $68 \%$, uso de botellas rellenables de $44 \%$ a $64 \%$, uso de bolsas de papel de un $19 \%$ a $57 \%$ y, uso de un termo de metal de un $17 \%$ a un $50 \%{ }^{2}$.

Con respecto al perfil del consumidor verde, Castañeda (2014), enfatiza que la población joven universitaria posee una mayor conciencia ecológica, siendo más sensible a los temas ecológicos y, por lo tanto, más propensa a actuar en pro del medio ambiente (Wang y cols., 2019). Por esta razón, Su y cols. (2019), consideraron que la generación de jóvenes nacidos después de 1995 experimentaría un mundo muy diferente en comparación con las generaciones anteriores, porque actualmente es más educada, experta en tecnología y orientada al bien de la comunidad. Asimismo, estos jóvenes tienen un fuerte sentido de responsabilidad social y el $59 \%$ desea ser parte del cambio en el desarrollo sustentable (Su y cols., 2019).

\section{Modelo de la Teoría del Comportamiento Planeado}

Los componentes del modelo de la TCP son las actitudes, las normas subjetivas y el control de compra percibido (Ajzen, 1991). En cuanto a la actitud, es considerada como una evaluación negativa o positiva que se hace sobre un comportamiento determinado considerando sus consecuencias; además, es considerada como el predictor principal de la intención conductual (Paul y cols., 2016) a critical variable in green marketing literature, intending to achieve triple bottom line (TBL. De igual manera, la actitud es una emoción encaminada a través de las evaluaciones de los individuos, es decir, si se tiene una actitud positiva hacia algún evento, las intenciones de comportamiento tienden a ser más positivas y viceversa (Chen y Tung, 2014). Además, una actitud es formada en la primera etapa del proceso de decisión de compra del consumidor (reconocimiento de la necesidad), puesto que, está influenciada por elementos como la información, naturaleza del producto, redes sociales, anuncios y otros factores de comportamiento (Pérez y cols., 2019).

En el contexto de productos verdes, Paul y cols. (2016)a critical variable in green marketing literature, intending to achieve triple bottom line (TBL, exponen que existe una relación positiva entre la actitud y la intención conductual a través de diversas culturas; por ejemplo, Birgelen y cols. (2009), señalaron que las personas que tienen una actitud positiva hacia el cuidado del medio ambiente, tienen una mayor preferencia hacia los productos envasados con materiales ecológicos, al uso de los servicios de hoteles ecológicos (Barber y cols., 2010), y a la adquisición de comida orgánica (Chen y Tung, 2014), entre otros.

\footnotetext{
1. Obtenido de: https://www.20minutos.com.mx/noticia/203141/0/mexicanos-consumen-cada-vez-mas-productos-amigables-con-el-medioambiente/

2. Obtenido de: https://www.kantarworldpanel.com/mx/Noticias-/Mas-mexicanos-toman-acciones-para-cuidar-medio-ambiente
} 
En tanto, las normas subjetivas son una función de las creencias normativas personales de un individuo y su motivación para aplicarlas (Chen, 2020), además, depende de la presión que siente una persona de realizar o no una acción, en la cual la opinión de los demás influye en sus decisiones (Prakash y Pathak, 2017). En otras palabras, el comportamiento de una persona es influenciado por la aprobación de amigos, familiares, compañeros de trabajo o socios comerciales (He y cols., 2019). De acuerdo con Prakash y Pathak (2017), las normas subjetivas dictan lo que moralmente es correcto o incorrecto; pero cuando se incorporan a un sistema de valores personales, se les conoce como normas personales, las cuales se describen como los sentimientos de una fuerte obligación moral de participar en actividades altruistas o ecológicas, sobre todo para los consumidores jóvenes. Por ejemplo, Wansink y cols. (2017), demostraron que la normas subjetivas afectaban el comportamiento de compra de productos amigables con el medio ambiente, cuando se mencionaba la existencia de grupos activistas, tales como: Friends of the Earth International ${ }^{3}$ y Greenpeace ${ }^{4}$. Asimismo, Jansson (2011), menciona que existe evidencia que las normas subjetivas y la intención conductual están positivamente correlacionadas en contextos de reciclaje y reducción de desperdicios (Li, Li, Cao y Niu, 2018; Matthies, Selge y Klöckner, en visitas a hoteles verdes (Teng y cols., 2015), uso de tecnología (Baker, Gahtani y Hubona, 2007), compra de productos orgánicos (Dean, Raats y Shepherd, 2012), consumo de verduras (Nystrand y Olsen, 2020)with an extension of self-efficacy and descriptive norms and, as well, hedonic and utilitarian eating values, is used as a conceptual framework. Structural equation modeling (SEM y, en estudios de predicción sobre consumir productos saludables versus productos no saludables (Burger y cols., 2010).

En cuanto al control de compra percibido, nos referimos a la facilidad o dificultad que un individuo presenta al momento de pretender realizar cierto comportamiento, poniendo de manifiesto las experiencias pasadas (Ajzen, 1991). Es evidente, entonces, que un individuo mostrará su intención de realizar cierto comportamiento al tener control para ejecutar el mismo (Yang y cols., 2003).
Por consiguiente, el control de compra percibido afecta directamente a las intenciones de realizar un comportamiento específico, debido a que se compone de dos factores, a saber: 1) las creencias de control, relacionadas con el sentido de la auto disponibilidad de habilidades, recursos y oportunidades; y 2) la facilidad de realizar lo descrito anteriormente (Baker y cols., 2007).

Dicho lo anterior, se espera que aquellas personas que perciben más control en su comportamiento tengan más intención de comprar (Ajzen, 1991). Por esto, el control de compra percibido ha sido estudiado en temas de reciclaje (Taylor y Todd, 1995), comidas orgánicas (Thøgersen, 2010) y en compras de productos verdes (Moser, 2015), por mencionar algunos.

A partir de las consideraciones anteriores, se propusieron las siguientes hipótesis:

H1: La actitud hacia la compra de productos verdes tiene un efecto positivo hacia la intención de compra de productos verdes.

H2: Las normas subjetivas hacia la compra de productos verdes tiene un efecto positivo hacia la intención de compra de productos verdes.

H3: El control de compra percibido hacia la compra de productos verdes tiene un efecto positivo hacia la intención de compra de productos verdes.

\section{La obligación moral y la intención de compra}

La obligación moral se presenta en el momento en que una persona participa en un acto ético sintiéndose orgulloso o culpable (Fu y cols., 2019). No obstante, Schwartz (1977), introdujo por primera vez el concepto de obligación personal conceptualizándola como un sentimiento individual de obligación moral para realizar ciertas formas de comportamiento prosocial, realzando su impacto en la predisposición de un individuo a ayudar a otro individuo.

Por otra parte, Chen (2016), describió la obligación moral como una variable importante para comprender a una persona que posee la intención de proteger al ambiente y de realizar un consumo de

3. "Amigos de la Tierra" fue fundado en 1969 por un grupo de activistas antinucleares. Sus campañas van más allá del tradicional movimiento de conservación y buscar para dirigir políticas económicas y aspectos de desarrollo de sostenibilidad. Obtenido de:_https://www.foei.org/es

4. Greenpeace es una ONG ambientalista, realiza campañas en todo el mundo por temas como la agricultura ecológica, los bosques, el cambio climático, contra el consumismo, por la democracia y el contrapoder, el desarme y la paz y el cuidado de los océanos. Obtenido de: https://www. greenpeace.orgl 
productos verdes. Además, Nguyen y cols. (2016) mencionaron que personas de culturas colectivistas tienden a considerar un juicio moral al tomar decisiones, de modo que prestan más atención a las normas que a las actitudes. Asimismo, Mazar y Zhong (2010), mencionan que las personas tienden a poseer un yo moral positivo; pero el poder mantenerlo implica confrontar dilemas sociales y éticos, los cuales las motivan a realizar acciones prosociales al ser amenazado su ser moral. Y, de la misma manera, Beldad y Hegner (2018), señalan que el consumo ético emerge de consideraciones morales, por lo que un consumidor ético que se siente fuertemente responsable hacia la sociedad comprará productos verdes. Por ejemplo, cuando una persona piensa que es su deber comprar productos amigables con el medio ambiente, porque de no hacerlo se sentirá culpable (Si y cols., 2020). Es evidente, entonces, la propuesta de la siguiente hipótesis:

H4: La obligación moral hacia la compra de productos verdes tiene un efecto positivo hacia la intención de compra de productos verdes.

En la figura 1 se muestra el modelo de estudio propuesto y las hipótesis desarrolladas previamente.

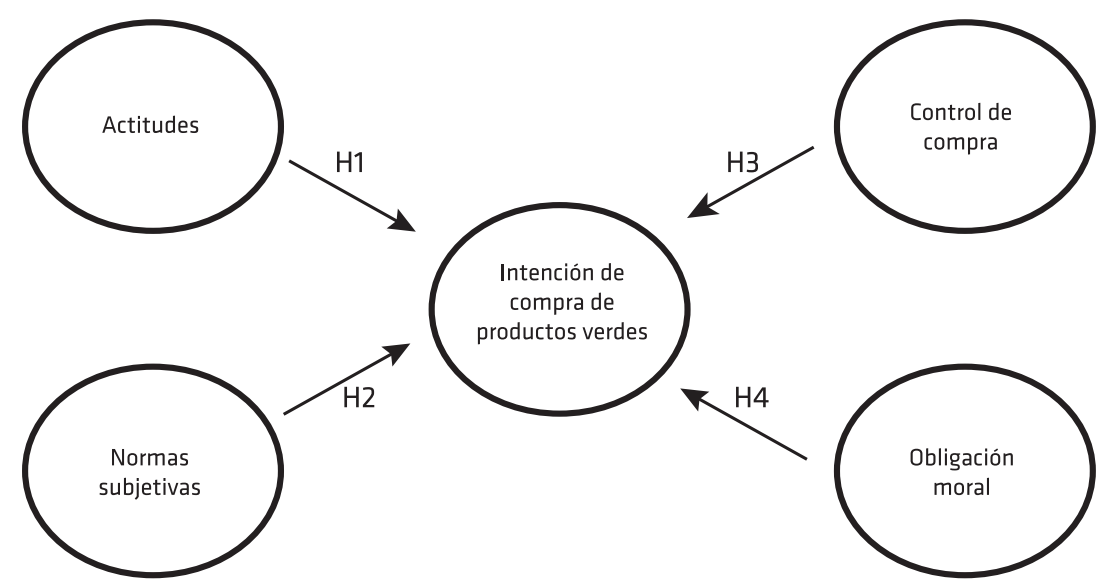

Figura 1. Modelo de estudio. Fuente: elaboración propia.

\section{METODOLOCIAA}

\section{Obtención de datos}

La obtención de datos se obtuvo a través de una encuesta en línea, la cual permite la replicabilidad y fortalece el poder estadístico (Khalifa y Liu, 2007; Wang y cols., 2019), a través de Formularios de Google (drive.google.com) ${ }^{5}$. La ventaja de realizar encuestas en línea es el bajo costo, permite la retroalimentación de manera más rápida, se tiene una mejor cobertura, se realiza en menor tiempo, y permite al investigador contactar de manera más rápida al grupo muestral (Sun y cols., 2020). Todos los ítems de medición fueron adoptados de estudios relacionados con el tema, validados previamente. Antes de iniciar la recolección de datos, la encuesta fue enviada a tres candidatos a
Doctor, quienes aportaron sus conocimientos para mejorar tanto la redacción de los ítems como el formato de la encuesta; posteriormente se envió a dos catedráticos con experiencia en temas de marketing verde, quienes dieron su aprobación para la aplicación de este.

Al inicio de la encuesta se presentó una lista de productos amigables con el medio ambiente que se ofrecen en el mercado, luego, el encuestado eligió todos aquellos productos verdes que ha comprado o utilizado por lo menos una vez. Lo anterior, con el fin de identificar qué tan relacionados están los encuestados con el uso actual de productos verdes (ver en apéndice).

En la segunda parte del cuestionario se midieron los ítems que forman los constructos de actitud hacia los productos verdes, normas subjetivas, ni-

5. https://forms.gle/m5gaD1GDQqMDM9Hm7 
vel de control de compra percibido, obligación moral y, finalmente, la intención de comprar productos verdes. Se aplicó una escala Likert de 7 puntos anclada, en donde 1 es "Totalmente en desacuerdo" y 7 es "Totalmente de acuerdo". En la Tabla 1 se presenta en detalle la operacionalización de las variables que se utilizaron en la encuesta. Para terminar, se solicitaron algunos datos demográficos como edad, nivel educativo y sexo (Ver Tabla 2).

El tipo de muestreo empleado fue no probabilístico, aplicando la técnica por conveniencia (Hair y cols., 2010). Además, el muestreo por conveniencia ha sido ampliamente utilizado en estudios relacionados al cuidado del medio ambiente, porque cumple con los criterios de facilidad de acceso, proximidad geográfica, disponibilidad del tiempo y voluntad de participar (Etikan, 2016; Janska y cols., 2020; Simon y cols., 2017; Sun y cols., 2020).

La muestra fue conformada por 280 alumnos de una universidad pública del estado de Tamaulipas, todos ellos estudiantes activos a nivel de licenciatura y postgrado de edades entre 18 y 40 años, cuyos correos obtuvimos a través de la base de datos que algunos maestros de la misma universidad nos proporcionaron. La incorporación de estudiantes como sujetos de análisis ha sido cuestionada y considerada como una fuente potencial de sesgo. No obstante, Exadaktylos y cols. (2013), han demostrado que estos no se comportan diferente de una población compuesta por participantes distintos a estudiantes (no-estudiantes). Además, el uso de muestras conformadas por estudiantes tiene amplias ventajas; una de ellas es la relacionada con la homogeneidad, porque se ha identificado que los estudiantes constituyen un grupo homogéneo que conduce a predicciones teóricas de mayor precisión que si se utilizaran muestras heterogéneas (Calder y cols., 1981).

El total de correos electrónicos enviados fue de 320 , de los cuales se obtuvo respuesta de 280 alumnos. La recopilación de datos se realizó del 29 de marzo al 16 de junio del año 2020.

En el grupo de encuestados, el 61.1\% corresponde a mujeres y el $38.9 \%$ a hombres. En lo que respecta a la edad, el $60.7 \%$ entre 18 y 24 años, el $28.2 \%$ entre 33 y 40 años, el $10.7 \%$ entre 25 y 32 $\mathrm{y}$, finalmente, el $0.4 \%$ corresponde a menores de 18 años. Con respecto al nivel educativo, el grupo más grande de encuestados (77.5\%) tenía el grado de licenciatura, seguido del nivel de maestría (16.4\%), así como de nivel doctorado (5.4\%) y, por último, en menor porcentaje otro grado diferente a los mencionados anteriormente (0.7\%). En la tabla 2 se presentan los resultados demográficos

\section{Especificación del Modelo}

Se decidió usar un modelo PLS-SEM, debido a que se busca identificar los constructos que afectan la intención de compra, además, porque en el modelo de medida se incluyen constructos formativos y reflectivos (Hair y cols., 2019).

De acuerdo con Hair y cols. (2019), la evaluación de Ios modelos PLS-SEM tiene dos etapas: la evaluación de los modelos de medida y la evaluación del modelo estructural. En cuanto a la evaluación de modelos de medida, primeramente, se especifica que los constructos de actitud, obligación moral e intención de compra son modelos de medida reflectiva, pues los ítems tienen una orientación de identificar posibles efectos de los constructos. Además, siguiendo las recomendaciones de Aldas y Uriel (2017), se calculó la correlación de los ítems por constructo, por lo tanto, presenta una correlación fuerte y positiva como se esperaba. Por su parte, los constructos de control de compra y normas subjetivas son modelos de medida formativa, porque los ítems fueron elaborados para identificar causas que formen el constructo y, en este caso, la correlación de sus ítems fue baja.

Por lo tanto, se siguieron las recomendaciones de Aldas y Uriel (2017) y Hair y cols. (2019), en la identificación de modelos de medida, su evaluación y la evaluación del modelo estructural. Para los constructos reflectivos, se requirió validar los constructos que tuvieran fiabilidad de consistencia interna - fiabilidad compuesta (CR) y un Alfa de Cronbach entre 0.60-0.90, una validez convergente - carga factorial mayor a 0.70 , comunalidad mayor a 0.50 y varianza extraída promedio (AVE) mayor 0.50 - y, validez discriminante utilizando el criterio de Fornell y Lacker (1981). Para la validación de constructos con medida formativa se realizó una validación convergente - cargas externa mayores a 0.50-, examinando que el grado de colinealidad - VIF sea menor a 5- y, establecer la significancia de los indicadores, es decir, que el intervalo de confianza no contiene el cero. En el modelo estructural se validó la capacidad predictiva del modelo mediante el coeficiente $R^{2}$ y $\underline{Q}^{2}$, el tamaño del efecto $f^{2}$ y, por último, se evaluó que las relaciones del modelo estructural fueran significativas. Se emplearon las paqueterías plsm (Sanchez y cols., 2013), matrixpls (Rönkkö y cols., 2020) y, pwr (Champely, 2020) del software estadístico RStudio para realizar las estimaciones requeridas. 
Tabla 1. Operacionalización de las variables relacionadas con la intención de compra de productos verdes

\begin{tabular}{|c|c|c|}
\hline Variables & Ítem & Referencias \\
\hline \multicolumn{3}{|l|}{ Actitudes } \\
\hline $\mathrm{AC1}$ & Comprar productos ecológicos es bueno. & Beldad y Hegner, (2018); Wang y cols. (2020); Yadav y Pathak (2016, \\
\hline $\mathrm{AC2}$ & $\begin{array}{l}\text { Al comprar un producto ecológico, yo podría marcar la diferen- } \\
\text { cia en el cuidado del medio ambiente. }\end{array}$ & 2017) \\
\hline $\mathrm{AC3}$ & Me gusta la idea de comprar productos ecológicos. & \\
\hline$A C 4$ & $\begin{array}{l}\text { Estoy de acuerdo que al comprar productos ecológicos cuida- } \\
\text { mos del medio ambiente. }\end{array}$ & \\
\hline \multicolumn{3}{|c|}{ Normas subjetivas } \\
\hline NS1 & $\begin{array}{l}\text { Mi familia influye fuertemente en mis decisiones de compra } \\
\text { de productos ecológicos. }\end{array}$ & Beldad y Hegner, (2018); Testa y cols. (2019); Wang y cols. (2016) \\
\hline NS2 & $\begin{array}{l}\text { Mis compañeros de escuela/trabajo influyen en mis deci- } \\
\text { siones de compra de productos ecológicos. }\end{array}$ & \\
\hline NS3 & $\begin{array}{l}\text { Las redes sociales influyen fuertemente en mis decisiones de } \\
\text { compra de productos ecológicos. }\end{array}$ & \\
\hline NS4 & $\begin{array}{l}\text { Creo que la mayoría de las personas que conozco recomiendan } \\
\text { la compra de productos ecológicos. }\end{array}$ & \\
\hline \multicolumn{3}{|c|}{ Control de compra percibido } \\
\hline CCP1 & Si decido comprar un producto ecológico, es mi decisión. & \\
\hline ССР2 & $\begin{array}{l}\text { Tengo la capacidad económica para comprar un producto } \\
\text { ecológico. }\end{array}$ & Beldad y Hegner (2018); Testa y cols. (2019); Wang y cols. (2020) \\
\hline ССРЗ & $\begin{array}{l}\text { Identifico fácilmente un producto ecológico de uno que NO } \\
\text { lo es. }\end{array}$ & \\
\hline ССР4 & $\begin{array}{l}\text { En mi opinión, los precios de los productos ecológicos NO son } \\
\text { altos. }\end{array}$ & \\
\hline \multicolumn{3}{|c|}{ Obligación moral } \\
\hline OM1 & $\begin{array}{l}\text { Usar productos ecológicos para cuidar del medio ambiente } \\
\text { está dentro de mis principios. }\end{array}$ & Diddi y Niehm (2017); Wang y cols. (2016); Yadav y Pathak (2016) \\
\hline $\mathrm{OM} 2$ & $\begin{array}{l}\text { Es mi deber moral comprar productos ecológicos siempre que } \\
\text { estén disponibles. }\end{array}$ & \\
\hline OM3 & $\begin{array}{l}\text { Comprar productos ecológicos me hace sentir que soy una } \\
\text { mejor persona. }\end{array}$ & \\
\hline OM4 & $\begin{array}{l}\text { Si no compro productos ecológicos, me sentiré culpable de no } \\
\text { cuidar del medio ambiente. }\end{array}$ & \\
\hline \multicolumn{3}{|c|}{ Intención de compra } \\
\hline IC1 & $\begin{array}{l}\text { Compraré productos ecológicos, aun cuando los productos que } \\
\text { no son ecológicos sean más baratos. }\end{array}$ & Beldad y Hegner (2018); Nystrand y Olsen (2020) (Sultan y cols., 2020) \\
\hline IC2 & $\begin{array}{l}\text { Si tuviera que comprar un producto hoy, compraría uno } \\
\text { ecológico. }\end{array}$ & \\
\hline IC3 & Planeo comprar productos ecológicos en el futuro. & \\
\hline IC4 & $\begin{array}{l}\text { Planeo cambiar la compra de mis productos por marcas que } \\
\text { sean amigables con el cuidado del medio ambiente. }\end{array}$ & \\
\hline
\end{tabular}

Fuente: elaboración propia.

Tabla 2. Perfil demográfico

\begin{tabular}{|c|c|c|}
\hline Datos demográficos & Frecuencia & Porcentaje \\
\hline \multicolumn{3}{|l|}{ Sexo } \\
\hline 1. Hombre & 109 & $38.9 \%$ \\
\hline 2. Mujer & 171 & $61.1 \%$ \\
\hline \multicolumn{3}{|l|}{ Edad } \\
\hline 1. Menos de 18 & 1 & $0.4 \%$ \\
\hline 2.19 a 24 & 170 & $60.7 \%$ \\
\hline 3. 25 a 32 & 30 & $10.7 \%$ \\
\hline 4. 33 a 40 & 79 & $28.2 \%$ \\
\hline \multicolumn{3}{|l|}{ Nivel educativo } \\
\hline \multicolumn{3}{|l|}{ 1. Licenciatura } \\
\hline 2. Maestría & 217 & $77.5 \%$ \\
\hline 3. Postgrado & 46 & $16.4 \%$ \\
\hline \multirow[t]{2}{*}{ 4. Otro } & 15 & $5.4 \%$ \\
\hline & 2 & $0.7 \%$ \\
\hline
\end{tabular}

Fuente: elaboración propia.

\section{RESULTADOS}

Los constructos reflectivos de actitud, obligación moral e intención de compra mostraron fiabilidad y validez en la medición que realizan, porque to- dos los constructos tienen consistencia interna y cumplieron con los criterios de validez (Hair y cols., 2019). Ver tabla 3. 
Tabla 3. Evaluación de los modelos de medida reflectiva

\begin{tabular}{|c|c|c|c|c|c|c|c|}
\hline \multirow{3}{*}{ Constructos } & \multirow{3}{*}{ İtems } & \multicolumn{3}{|c|}{ Validez Convergente } & \multicolumn{2}{|c|}{ Fiabilidad de consistencia interna } & \multirow{2}{*}{ Validez discriminante } \\
\hline & & Cargas & Comunalidad & AVE & $\begin{array}{l}\text { Fiabilidad } \\
\text { Compuesta }\end{array}$ & Alfa de Cronbach & \\
\hline & & & & & & & $\begin{array}{c}\text { Cumple criterio de Fornell y } \\
\text { Larcker }\end{array}$ \\
\hline \multirow{4}{*}{ Actitud } & AC1 & 0.81 & 0.66 & \multirow{4}{*}{0.75} & \multirow{4}{*}{0.92} & \multirow{4}{*}{0.89} & \multirow{4}{*}{$\mathrm{Si}$} \\
\hline & $\mathrm{AC2}$ & 0.85 & 0.72 & & & & \\
\hline & AC3 & 088 & 078 & & & & \\
\hline & AC4 & 0.91 & 0.82 & & & & \\
\hline \multirow{5}{*}{ Obligación } & OBM1 & 0.82 & 0.68 & \multirow{5}{*}{0.61} & \multirow{5}{*}{0.86} & \multirow{5}{*}{0.79} & \multirow{5}{*}{$\mathrm{Si}$} \\
\hline & OBM2 & 0.87 & 0.75 & & & & \\
\hline & & & & & & & \\
\hline & ОВм3 & 0.73 & 0.54 & & & & \\
\hline & OBM4 & 0.70 & 0.48 & & & & \\
\hline \multirow{4}{*}{$\begin{array}{l}\text { Intención de } \\
\text { Compra }\end{array}$} & IC1 & 0.78 & 0.61 & \multirow{4}{*}{0.63} & \multirow{4}{*}{0.88} & \multirow{4}{*}{0.81} & \multirow{4}{*}{$\mathrm{Si}$} \\
\hline & IC2 & 0.88 & 0.77 & & & & \\
\hline & IC3 & 0.78 & 0.60 & & & & \\
\hline & IC4 & 0.74 & 0.55 & & & & \\
\hline
\end{tabular}

Fuente: elaboración propia.

Para la validación de los constructos con medida formativa se estimó el modelo bootstrap con 5000 muestras, únicamente el ítem CCP1 del constructo de control de compra no fue significativo, por lo que se decidió eliminar y se descartó colinealidad entre los ítems (Tabla 4). Al volver a realizar la validación al constructo de control de compra se cumplió la validez convergente (Tabla 5).

Tabla 4. Evaluación de los modelos de medida formativa

\begin{tabular}{|c|c|c|c|c|c|c|}
\hline Constructo & Ítems & Pesos externos & Cargas externas & Intervalo de confianza $95 \%$ & VIF & Carga significativa \\
\hline \multirow{4}{*}{ Control } & CCP1 & 0.23 & 0.43 & {$\left[\begin{array}{lll}-0.06 & 0.78\end{array}\right]$} & 1.11 & No \\
\hline & CCP2 & 0.36 & 0.70 & {$[0.350 .86]$} & 1.25 & Sí \\
\hline & ССР3 & 0.47 & 0.74 & {$\left[\begin{array}{lll}0.37 & 0.92\end{array}\right]$} & 1.16 & Sí \\
\hline & СCP4 & 0.44 & 0.69 & {$[0.290 .88]$} & 1.18 & Sí \\
\hline \multirow{4}{*}{ Normas } & NS1 & 0.42 & 0.64 & {$\left[\begin{array}{lll}0.26 & 0.84\end{array}\right]$} & 1.57 & Sí \\
\hline & NS2 & -0.02 & 0.58 & [0.19 0.81] & 2.06 & Sí \\
\hline & NS3 & 0.07 & 0.47 & {$\left[\begin{array}{lll}0.06 & 0.74\end{array}\right]$} & 1.52 & Sí \\
\hline & NS4 & 0.79 & 0.91 & {$\left[\begin{array}{lll}0.66 & 0.98\end{array}\right]$} & 1.20 & Sí \\
\hline
\end{tabular}

Fuente: elaboración propia. 
Tabla 5. Evaluación corregida de los modelos de medida formativa

\begin{tabular}{|c|c|c|c|c|c|c|}
\hline Constructo & Ítems & Pesos externos & Cargas externas & Intervalo de confianza 95\% & VIF & Carga significativa \\
\hline \multirow{3}{*}{ Control } & CCP2 & 0.43 & 0.72 & {$\left[\begin{array}{lll}0.40 & 0.90\end{array}\right]$} & 1.19 & Sí \\
\hline & ССР3 & 0.52 & 0.75 & {$\left[\begin{array}{lll}0.42 & 0.95\end{array}\right]$} & 1.13 & Sí \\
\hline & CCP4 & 0.42 & 0.70 & {$\left[\begin{array}{ll}0.33 & 0.93\end{array}\right]$} & 1.17 & Sí \\
\hline \multirow{4}{*}{ Normas } & NS1 & 0.42 & 0.64 & {$\left[\begin{array}{lll}0.26 & 0.84\end{array}\right]$} & 1.57 & Sí \\
\hline & NS2 & -0.02 & 0.58 & {$\left[\begin{array}{lll}0.19 & 0.81]\end{array}\right]$} & 2.07 & Sí \\
\hline & NS3 & 0.07 & 0.47 & {$\left[\begin{array}{lll}0.06 & 0.74\end{array}\right]$} & 1.52 & Sí \\
\hline & NS4 & 0.79 & 0.91 & {$\left[\begin{array}{lll}0.66 & 0.98\end{array}\right]$} & 1.20 & Sí \\
\hline
\end{tabular}

Fuente: elaboración propia.

Al evaluar el modelo estructural se encontró que la actitud y las normas subjetivas no tienen una relación significativa hacia la compra de productos verdes (el intervalo contiene el cero), por lo que no fue posible confirmar las hipótesis 1 y 2 . Con respecto a las hipótesis 3 y 4 , se pudieron confirmar, puesto que el control de compra y la obligación moral tiene un efecto positivo y significativo hacia la intención de compra de productos verdes. Además, se pudo apreciar que la obligación moral tiene un mayor efecto sobre la intención de compra (Tabla 6).

Tabla 6. Significancia de las relaciones estructurales

\begin{tabular}{lcccc}
\multicolumn{1}{c}{ Constructos } & Original & Mean.Boot & Std.Error & perc.025 \\
\hline Actitud $\rightarrow$ Int_compra & 0.05 & 0.05 & 0.05 & -0.04 \\
Control $\rightarrow$ Int_compra & 0.12 & 0.13 & 0.06 & 0.01 \\
Obligación $\rightarrow$ Int_compra & 0.59 & 0.58 & 0.05 & 0.47 \\
Normas $\rightarrow$ Int_compra & -0.01 & 0.01 & 0.06 & -0.11 \\
\hline
\end{tabular}

Fuente: elaboración propia.

Finalmente, el modelo puede explicar moderadamente la intención de compra, porque el es de 0.43 con respecto al efecto en tamaño, siendo también moderado, dado que el es de 0.05 y, visto que todos los son positivos, el modelo presenta una relevancia predictiva (Tabla 7).

Tabla 7. Valores de $\mathrm{Q}^{2}$

\begin{tabular}{ccccc}
\hline Actitud & Control & Obligación & Normas & Intención de compra \\
\hline 0.70 & 0.11 & 0.33 & 0.43 \\
\hline
\end{tabular}

Fuente: elaboración propia.

\section{CONCLUSIONES}

Como se mencionó previamente, el estudio tiene por objetivo identificar qué variables del modelo de la Teoría del Comportamiento Planeado realmente afectan en la intención de compra de productos verdes en los jóvenes del noreste de
México y, además, demostrar que la variable de obligación moral debe incluirse en dicho modelo. A partir de los resultados obtenidos, se pudo constatar que únicamente las variables significativas del modelo propuesto fueron el control de compra percibido (H3) y la obligación moral (H4). 
Estudios previos sobre la variable de control de compra percibido, determinaron que tiene la mayor influencia significativa en la intención de compra del consumidor (Paul y cols., 2016; Simsekoglu y Nayum, 2019; Taufique y Vaithianathan, 2018; Xu y cols., 2020)great attention has been paid to green furniture. This study attempts to explore consumer's intention to purchase green furniture by applying an extension model of theory of planned behavior (TPB y, en muchas investigaciones sus resultados se basan en la disponibilidad del producto (Xu y cols., 2020), facilidad de compra (Simsekoglu y Nayum, 2019), compras pasadas (Carfora y cols., 2017) y, variedad de productos (Paul y cols., 2016). Sin embargo, en estudios previos no tuvo la misma influencia significativa como en el presente estudio (Icek Ajzen, 2015; Yarimoglu y Gunay, 2020; Yazdanpanah y Forouzani, 2015).

En cuanto a la obligación moral, Spielmann (2020), menciona que el $70 \%$ de los consumidores siente que es su responsabilidad estar comprometidos en comportamientos más verdes, provocando que se sientan satisfechos y compren marcas vinculadas a causas prosociales. De igual manera, Chan y Bishop (2013), extendieron el modelo de la Teoría del Comportamiento Planeado con la variable de obligación moral y, los resultados constataron que dicha variable se asociaba con una mayor intención hacia actividades pro sociales, como el reciclaje. Esto refleja la importancia de las normas morales como fuertes impulsores de comportamientos proambientales en comparación con la normas subjetivas (Issock y cols., 2020).

En cuanto al constructo de actitud, este no presentó una relación significativa hacia la compra de productos verdes. De manera semejante, Xu y cols. (2020), expusieron que la actitud de los jóvenes consumidores chinos entre 20 y 40 años de edad no está significativamente relacionada con la intención de compra de productos verdes, lo que implica una brecha entre la actitud y el comportamiento ecológico. Sin embargo, existen estudios previos que confirman que la actitud de los consumidores jóvenes en el consumo de productos verdes en otros países es significativa (Asif y cols., 2018; Chekima y cols., 2016; Huang y Ce, 2019; Taufique y Vaithianathan, 2018). Dicho esto, es importante considerar que existe el supuesto de que los consumidores jóvenes presentan una conducta humana totalmente racional, basándose en el uso de la información disponible (Castañeda, 2014), por lo que hay que seguir trabajan- do con campañas publicitarias que induzcan a la compra de productos verdes en este segmento de mercado (Si y cols., 2020)especially dockless bike sharing (DBS, proporcionándoles conocimiento del problema ambiental y, por sobre todo, considerar que es posible encontrar diferencias en la intención conductual de un país a otro (Asif y cols., 2018).

De igual manera, las normas subjetivas no presentaron una relación significativa hacia la compra de productos verdes. Dicho resultado, coincide con lo expuesto por Paul y cols. (2016)a critical variable in green marketing literature, intending to achieve triple bottom line ( $T B L$, demostrando que las normas subjetivas en el consumidor de la India no son un predictor importante para la intención de compra, en tal sentido, no sienten la necesitan de la aprobación de otras personas importantes para comprar productos verdes. De igual forma, Xu y cols. (2020), determinaron que las normas subjetivas no tienen influencia significativa en la intención de compra de muebles ecológicos auténticos en China; lo anterior, como resultado de que los consumidores no estaban seguros si sus amigos o familiares querían que compraran productos verdes; aunque, la variable de normas subjetivas ha sido relacionada de manera positiva con la intención de compra de productos verdes (Burger y cols., 2010; Chen y Tung, 2010; Dean y cols., 2012; Pickett-Baker y Ozaki, 2008; Teng y cols., 2015)

\section{Limitaciones}

La presente investigación presenta algunas limitaciones que pueden ser abordadas en futuras investigaciones. Primero, la investigación se realizó en una región del Noreste de México, la cual puede tener diferencias significativas en otras regiones del mismo territorio nacional; además, se contó con una muestra pequeña y, en cambio, una muestra más grande agregaría más influencia a las conclusiones presentadas (Spielmann, 2020). Segundo, se pueden realizar estudios comparativos entre diferentes grupos generacionales con diferentes perfiles demográficos, como edad, nivel educativo, ocupación e ingresos. Tercero, se pueden llevar a cabo estudios longitudinales para examinar los cambios en cada una de las variables del modelo; así como, la forma en que sus intenciones se convierten en un real comportamiento de compra, por ejemplo: antes y después del COVID-19. Y, finalmente, el estudio se enfocó en medir la intención de compra de productos verdes, lo 
que nos permite ampliar la investigación en otras categorías de productos o actividades proambientales, como compra de alimentos orgánicos, reutilización de productos, cuidado del agua, actividades de reforestación, reciclaje, entre otros.

\section{APÉNDICE}

Parte I. Instrucciones: antes de iniciar el cuestionario se presenta una lista de productos amigables con el medio ambiente que se ofrecen en el mercado, selecciona todos aquellos que actualmente has comprado o que has utilizado, por lo menos en una ocasión.

Bolsas ecológicas de supermercado

Popotes de acero

Focos ahorradores

Bolsas de basura biodegradables

Maquillaje orgánico

Termos de acero

Cepillo de dientes de bambú

Cubiertos reutilizables

Ninguno

Otros

¿Cuáles?

\section{BIBLIOGRAFÍA}

Aldás Manzano, J. y Uriel Jiménez, E. (2017). Análisis multivariante aplicado con R. Ediciones Paraninfo.

Ajzen, I. (1991). The theory of planned behavior, Organ Behav Hum Decis Process, 50, 179-211.

Ajzen, Icek. (2002). Perceived behavioral control, self-efficacy, locus of control, and the theory of planned behavior. Journal of Applied Social Psychology, 32(4), 665-683.

Ajzen, Icek. (2015). The theory of planned behaviour is alive and well, and not ready to retire: a commentary on Sniehotta, Presseau, and Araújo-Soares. Health Psychology Review, 9(2), 131-137.
Asif, M., Xuhui, W., Nasiri, A. y Ayyub, S. (2018) Determinant factors influencing organic food purchase intention and the moderating role of awareness: A comparative analysis. Food Ouality and Preference, 63, 144-150.

Baker, E. W., Al-Gahtani, S. S. y Hubona, G. S (2007). The effects of gender and age on new technology implementation in a developing country: Testing the theory of planned behavior (TPB). Information Technology a People, 20 (4), 352-375

Barber, N., Taylor, D. C. y Deale, C. S. (2010). Wine tourism, environmental concerns, and purchase intention. Journal of Travel \& Tourism Marketing, 27(2), 146-165.

Beldad, A. y Hegner, S. (2018). Determinants of Fair Trade Product Purchase Intention of Dutch Consumers According to the Extended Theory of Planned Behaviour: The Moderating Role of Gender. Journal of Consumer Policy, 41(3), 191-210.

Burger, J. M., Bell, H., Harvey, K., Johnson, J., Stewart, C., Dorian, K. y Swedroe, M. (2010). or delicious? The effect of descriptive norm information on food Nutritiouschoice. Journal of Social and Clinical Psychology, 29(2), 228-242.

Calder, B. J., Phillips, L. W. y Tybout, A. M. (1981). Designing Research for Application. Journal of consumer research, 8(2), 197-207.

Carfora, V., Caso, D., Sparks, P., y Conner, M. (2017) Moderating effects of pro-environmental self-identity on pro-environmental intentions and behaviour: A multi-behaviour study. Journal of Environmental Psychology, 53, 92-99.

Castañeda, J. S. (2014). Contextualización y enfoques en el estudio de comportamientos proambientales o ecológicos con miras a la perfilación del consumidor verde. Suma de Negocios, 5(10), 34-39.

Champely, S. (2017). pwr: Basic functions for power analysis. R Package Version 1.2-1.

Chan, L. y Bishop, B. (2013a). A moral basis for recycling: Extending the theory of planned behaviour. Journal of Environmental Psychology, 36(1), 96-102. 
Chan, L. y Bishop, B. (2013b). A moral basis for recycling: Extending the theory of planned behaviour. Journal of Environmental Psychology, 36, 96-102.

Chekima, B. C., Syed Khalid Wafa, S. A. W., Igau, O. A., Chekima, S. y Sondoh, S. L. (2016). Examining green consumerism motivational drivers: Does premium price and demographics matter to green purchasing?. Journal of Cleaner Production, 112, 3436-3450.

Chen, M. F. (2016). Extending the theory of planned behavior model to explain people's energy savings and carbon reduction behavioral intentions to mitigate climate change in Taiwan-moral obligation matters. Journal of Cleaner Production, 112, 1746-1753.

Chen, M. F. (2020). The impacts of perceived moral obligation and sustainability self-identity on sustainability development: A theory of planned behavior purchase intention model of sustainability-labeled coffee and the moderating effect of climate change skepticism. Business Strategy and the Environment, 1-14.

Chen, M. F. y Tung, P. J. (2010). The moderating effect of perceived lack of facilities on consumers' recycling intentions. Environment and Behavior, 42(6), 824-844.

Chen, M. F. y Tung, P. J. (2014). Developing an extended Theory of Planned Behavior model to predict consumers' intention to visit green hotels. International journal of hospitality management, 36, 221-230.

Dean, M., Raats, M. M. y Shepherd, R. (2012). The Role of Self-Identity, Past Behavior, and Their Interaction in Predicting Intention to Purchase Fresh and Processed Organic Food. Journal of Applied Social Psychology, 42(3), 669-688.

Diddi, S. y Niehm, L. S. (2017). Exploring the role of values and norms towards consumers' intentions to patronize retail apparel brands engaged in corporate social responsibility (CSR). Fashion and Textiles, 4(1), 5.

Etikan, I. (2016). Comparison of Convenience Sampling and Purposive Sampling. American journal of theoretical and applied statistics, 5(1), 1-4.

Exadaktylos, F., Espín, A. M. y Brañas-Garza, P. (2013). Experimental subjects are not different. Scientific Reports, 3, 1-6.
F. Hair, J., P.Bush, R. y Ortinau, D. J. (2010). Investigación de mercados En un ambiente de información digital. México: McGraw-Hill

Fu, B., Kurisu, K., Hanaki, K. y Che, Y. (2019). Influential factors of public intention to improve the air quality in China. Journal of cleaner production, 209, 595-607.

Hair Jr., J. F., M. Hult, G. T., M. Ringle, C., Sarstedt, M., Castillo Apraiz, J., Cepeda Carrión, G. A. y Roldán, J. L. (2019). Manual de Partial Least Squares Structural Equation Modeling (PLSSEM) (Segunda Edición). In Manual de Partial Least Squares Structural Equation Modeling (PLS-SEM).

Han, H. y Kim, Y. (2010). An investigation of green hotel customers' decision formation: Developing an extended model of the theory of planned behavior. International journal of hospitality management, 29(4), 659-668.

He, H., Fu, J., Li, X. y Guo, R. (2019). The interplay between endorser social status and normative appeals on the endorsement effectiveness of pro-environmental behaviors. PLOS ONE, 14(1), 1-17.

Hong, Z., Wang, H. y Yu, Y. (2018). Green product pricing with non-green product reference. Transportation Research Part E: Logistics and Transportation Review, 115, 1-15.

Huang, X. y Ge, J. (2019). Electric vehicle development in Beijing: An analysis of consumer purchase intention. Journal of Cleaner Production, 216, 361-372.

Issock, P. B. I., Roberts-Lombard, M. y Mpinganjira, M. (2020). Normative Influence on Household Waste Separation: The Moderating Effect of Policy Implementation and Sociodemographic Variables. Social Marketing Quarterly, 26(2), 93-110.

Janska, M., Kollar, P. y Celer, C. (2020). Factors Influencing Consumption of Organic Food. Zagreb International Review of Economics o Business, 23(1), 81-94.

Jansson, J. (2011). Consumer eco-innovation adoption: Assessing attitudinal factors and perceived product characteristics. Business Strategy and the Environment, 20(3), 192-210.

Khalifa, M. y Liu, V. (2007). Online consumer retention: Contingent effects of online shopping habit and online shopping experience. European Journal of Information Systems, 16(6), 780-792. 
Larios-Gómez, E. (2019). Relation regarding among mexican ecological consumers. affect, concern and knowledge and purchase behavior: A study. Revista Brasileira de Marketing, 18(3), 73-100.

Li, J., Li, D., Cao, Q. y Niu, X. (2018). The role of regret and disappointment in the repurchase effect: Does gender matter?. Journal of Behavioral and Experimental Economics, 75(July 2017), 134-140.

Martins, J., Costa, C., Oliveira, T., Gonçalves, R. y Branco, F. (2019). How smartphone advertising influences consumers' purchase intention. Journal of Business Research, 94(August 2017), 378-387.

Matthies, E., Selge, S. y Klöckner, C. A. (2012). The role of parental behaviour for the development of behaviour specific environmental norms - The example of recycling and re-use behaviour. Journal of Environmental Psychology, 32(3), 277-284.

Mazar, N. y Zhong, C. B. (2010). Do green products make us better people?. Psychological Science, 21(4), 494-498.

Moser, A. K. (2015). Thinking green, buying green? Drivers of pro - Environmental purchasing behavior. Journal of Consumer Marketing, 32 (3), 167-175

Mostafa, M. M. (2006). Antecedents of Egyptian Consumers' Green Purchase Intentions. Journal of International Consumer Marketing, 19(2), 97-126.

Nguyen, T. K. C., Nguyen, D. M., Trinh, V. T., Tran, T. P. D. Y Cao, T. P. (2020). Factors Affecting Intention to Purchase Green Products in Vietnam. The Journal of Asian Finance, Economics and Business, 7(4), 205-211.

Nguyen, T. N., Lobo, A., Nguyen, H. L., Phan, T. T. H. y Cao, T. K. (2016). Determinants influencing conservation behaviour: Perceptions of Vietnamese consumers. Journal of Consumer Behaviour, 15(6), 560-570.

Nystrand, B. T. y Olsen, S. O. (2020). Consumers' attitudes and intentions toward consuming functional foods in Norway. Food Quality and Preference, 80, 103827.

Paul, J., Modi, A. y Patel, J. (2016a). Predicting green product consumption using theory of planned behavior and reasoned action. Journal of retailing and consumer services, 29, 123-134.
Paul, J., Modi, A. y Patel, J. (2016b). Predicting green product consumption using theory of planned behavior and reasoned action. Journal of Retailing and Consumer Services, 29, 123-134.

Pérez-Villarreal, H. H., Martínez-Ruiz, M. P. y Izquierdo-Yusta, A. (2019). Testing model of purchase intention for fast food in Mexico: How do consumers react to food values, positive anticipated emotions, attitude toward the brand, and attitude toward eating hamburgers?. Foods, 8(9), 369.

Pickett-Baker, J. y Ozaki, R. (2008). Pro-environmental products: Marketing influence on consumer purchase decision. Journal of Consumer Marketing, 25, 281-93

Prakash, G. y Pathak, P. (2017). Intention to buy eco-friendly packaged products among young consumers of India: A study on developing nation. Journal of Cleaner Production, 141, 385393.

Rashid, N. R. N. A. (2009). Awareness of Eco-label in Malaysia's Green Marketing Initiative. International Journal of Business and Management, 4(8), 132-141.

Rönkkö, M., Mclntosh, C. N. y Aguirre-Urreta, M. I. (2016). Improvements to PLSc: Remaining problems and simple solutions. Unpublished Working Paper.

Sanchez, G., Trinchera, L. y Russolillo, G. (2013). plspm: tools for partial least squares path modeling (PLS-PM). R Package version 0.4, 1.

Schill, M., Godefroit-Winkel, D., Diallo, M. F. y Barbarossa, C. (2019). Consumers' intentions to purchase smart home objects: Do environmental issues matter? Ecological Economics, 161, 176-185.

Schwartz, S. H. (1977). Normative influences on altruism. Advances in experimental social psychology, 10(1), 221-279.

Si, H., Shi, J. gang, Tang, D., Wu, G. y Lan, J. (2020). Understanding intention and behavior toward sustainable usage of bike sharing by extending the theory of planned behavior. Resources, Conservation and Recycling, 152, 104513.

Simon, L., Moraes, C. A. M., Modolo, R. C. E., Vargas, M., Calheiro, D. y Brehm, F. A. (2017). Recycling of contaminated metallic chip based on eco-efficiency and eco-effectiveness approaches. Journal of cleaner production, 153, 417-424. 
Simsekoglu, Ö. y Nayum, A. (2019). Predictors of intention to buy a battery electric vehicle among conventional car drivers. Transportation Research Part F: Traffic Psychology and Behaviour, 60, 1-10.

Spielmann, N. (2020). Green is the New White: How Virtue Motivates Green Product Purchase. Journal of Business Ethics, 1-18.

Su, C. H., Tsai, C. H., Chen, M. H. y Lv, W. Q. (2019). U.S. sustainable food market generation Z consumer segments. Sustainability, 11(13), 3607.

Sultan, P., Tarafder, T., Pearson, D. y Henryks, J. (2020). Intention-behaviour gap and perceived behavioural control-behaviour gap in theory of planned behaviour: moderating roles of communication, satisfaction and trust in organic food consumption. Food Quality and Preference, 81, 103838.

Sun, Y., Luo, B., Wang, S. y Fang, W. (2020). What you see is meaningful: Does green advertising change the intentions of consumers to purchase eco-labeled products? Business Strategy and the Environment, 1-11

Szabo, S. y Webster, J. (2020). Perceived Greenwashing: The Effects of Green Marketing on Environmental and Product Perceptions. Journal of Business Ethics, 1-21.

Taufique, K. M. R. y Vaithianathan, S. (2018). A fresh look at understanding Green consumer behavior among young urban Indian consumers through the lens of Theory of Planned Behavior. Journal of cleaner production, 183, 46-55.

Taylor, S. y Todd, P. (1995). An Integrated Model of Waste Management Behavior: A Test of Household Recycling and Composting Intentions. Environment and behavior, 27(5), 603630.

Teng, Y. M., Wu, K. S. y Liu, H. H. (2015). Integrating Altruism and the Theory of Planned Behavior to Predict Patronage Intention of a Green Hotel. Journal of Hospitality a Tourism Research, 39(3), 299-315.

Testa, F., Sarti, S. y Frey, M. (2019). Are green consumers really green? Exploring the factors behind the actual consumption of organic food products. Business Strategy and the Environment, 28(2), 327-338.
Thøgersen, J. (2010). Country differences in sustainable consumption: The case of organic food. Journal of Macromarketing, 30(2), 171185.

van Birgelen, M., Semeijn, J. y Keicher, M. (2009). Packaging and proenvironmental consumption Behavior: Investigating purchase and disposal decisions for beverages. Environment and Behavior, 41(1), 125-146.

Wang, H., Ma, B. y Bai, R. (2019). How Does Green Product Knowledge Effectively Promote Green Purchase Intention?. Sustainability, 11(4), 1193.

Wang, J., Tao, J. y Chu, M. (2020). Behind the label: Chinese consumers' trust in food certification and the effect of perceived quality on purchase intention. Food Control, 108, 106825.

Wang, L., Wong, P. P. W. y Narayanan, E. A. (2019). The demographic impact of consumer green purchase intention toward Green Hotel Selection in China. Tourism and Hospitality Research, 20(2), 210-222.

Wang, S., Fan, J., Zhao, D., Yang, S. y Fu, Y. (2016). Predicting consumers' intention to adopt hybrid electric vehicles: using an extended version of the theory of planned behavior model. Transportation, 43(1), 123-143.

Wang, Y. C. y Beise-Zee, R. (2013). Preencounter Affective States of Business Travelers and Service Responses. Journal of Hospitality Marketing and Management, 22(6), 634-655.

Wansink, B., Soman, D. y Herbst, K. C. (2017). Larger partitions lead to larger sales: Divided grocery carts alter purchase norms and increase sales. Journal of Business Research, 75, 202-209.

Xu, X., Hua, Y., Wang, S. y Xu, G. (2020). Determinants of consumer's intention to purchase authentic green furniture. Resources, Conservation and Recycling, 156, 104721.

Xu, X., Wang, S. y Yu, Y. (2020). Consumer's intention to purchase green furniture: Do health consciousness and environmental awareness matter?. Science of the Total Environment, 704, 135275.

Yadav, R. y Pathak, G. S. (2016). Intention to purchase organic food among young consumers: Evidences from a developing nation. Appetite, 96, 122-128. 
Yadav, R. y Pathak, G. S. (2017). Determinants of Consumers' Green Purchase Behavior in a Developing Nation: Applying and Extending the Theory of Planned Behavior. Ecological Economics, 134, 114-122.

Yang-Wallentin, F., Schmidt, P., Davidov, E. y Bamberg, S. (2003). Is there any interaction effect between intention and perceived behavioral control?. MPR-Online, 8(2), 127-157.

Yarimoglu, E. y Gunay, T. (2020). The extended theory of planned behavior in Turkish customers' intentions to visit green hotels. Business Strategy and the Environment, 29(3), 10971108.
Yazdanpanah, M. y Forouzani, M. (2015). Application of the Theory of Planned Behaviour to predict Iranian students' intention to purchase organic food. Journal of Cleaner Production, 107, 342-352.

Zhikun, D. y Fungfai, N. (2009). Knowledge sharing among architects in a project design team: An empirical test of theory of reasoned action in China. Chinese Management Studies, 3 (2), 130-142 
Revista Academia \& Negocios Vol.7 (1) 2021 pp. 15-30 